\title{
GEOARQUEOLOGÍA: INTERPRETACIONES INTERDISCIPLINARIAS PARA LA INVESTIGACIÓN ARQUEOLÓGICA
}

\author{
GEOARCHAEOLOGY: INTERDISCIPLINARY EXPLANATIONS FOR THE \\ ARCHAEOLOGICAL RESEARCH
}

\author{
Benjamín Acevedo \\ Escuela de Antropología, Universidad de Costa Rica, Ciudad Universitaria \\ Rodrigo Facio, San José, Costa Rica \\ benjamín.acevedo@ucr.ac.cr
}

(Recibido: 11/08/2014; aceptado: 09/09/2014)

\begin{abstract}
Geoarchaeological research involves interdisciplinary work. The objectives, theoretical framework, methodological approach and the final analysis are built together from archeology and disciplines within the earth sciences to better understand what life was like in the past. The Geological Journal of Central America is a journal to present joint work between geoscientists and archaeologists. Together these fields can present a unique approach to material remains, expanding the range of issues, analysis and interpretations thereby brining new light to some of the traditional methods and techniques in archaeology.

Keywords: Geoarchaeology, archaeological geology, archaeology, interdisciplinarity, Costa Rica.

RESUMEN: Las investigaciones geoarqueológicas implican la realización de trabajos interdisciplinarios, donde los objetivos, modelo teórico, estrategia metodológica y análisis se construyen conjuntamente desde la arqueología y disciplinas comprendidas por las ciencias de la tierra, para al final, llegar a la resolución de un problema concerniente a cómo fue la vida en el pasado. La Revista Geológica de América Central, viene a ser un medio para difundir los trabajos conjuntos entre geocientíficos y arqueólogos, quienes muestran una lectura diferente de los restos materiales, que vienen a ampliar la gama de temáticas, análisis e interpretaciones, que hasta el momento brindaban los métodos y técnicas tradicionales en arqueología.

Palabras clave: Geoarqueología, geología arqueológica, arqueología, interdisciplinariedad, Costa Rica.
\end{abstract}




\section{INTRODUCCIÓN}

Todo problema arqueológico inicia como un problema geoarqueológico, esta es una famosa frase del arqueólogo estadounidense Colin Renfrew (1976), que si bien puede estar bajo el hilo del cuestionamiento de algunas de las corrientes arqueológicas actuales, para los geocientíficos involucrados en cuestiones relativas a la historia del ser humano, viene a ser una bienvenida al campo de la arqueología.

La asociación de la investigación arqueológica con las características paleoambientales, es decir, en aquellas en las que se integra, información arqueológica, biológica y geológica, para conocer cómo fue que vivieron las personas en el pasado, se remonta a los trabajos pioneros de los daneses Jens Jacob Asmussen Worsaae, Johannes Japetus Smith Steenstrup y Johan Georg Forchhammer, en la década de 1830. Así como en Escandinavia, toda la información recabada, referente al retroceso de los glaciares, cambios del nivel del mar, así como en el clima, flora y fauna, brindaron a los arqueólogos información que facilitó una mejor comprensión del uso de la tierra por parte de las poblaciones antiguas (Trigger, 2006).

Este enfoque estimuló el estudio de paleoambientes, y si bien no constituían análisis de culturas completas, el interés creciente en las relaciones entre las sociedades humanas y su entorno ambiental, propició un punto de vista funcionalprocesual, de uno de los mayores aspectos del comportamiento humano, es decir, la adaptación de las culturas antiguas a condiciones ambientales pasadas (Trigger, 2006).

Así, durante el siglo XIX, las colaboraciones entre geocientíficos y arqueológos, empiezan a develar cuestiones relativas a paleoambientes, y ya para inicios del siglo XX, se comienzan a consolidar los estudios enfocados en la formación del registro arqueológico, establecimiento de cronologías y caracterización de artefactos (cerámicos y líticos) y ecofactos (elementos naturales no modificados, que brindan datos para conocer cómo fue la vida de poblaciones antiguas). Pero es hasta la década de 1970, cuando se da un incremento de la designación de una variedad de tipos de investigaciones, que usan técnicas propias de las geociencias, en la evaluación del registro arqueológico (Rapp \& Hill, 1998).

Como ya se ha mencionado, la geoarqueología no es un campo nuevo, sin embargo en nuestro contexto centroamericano, aún se puede considerar un espacio nada conocido, tanto para geólogos como arqueólogos. La Revista Geológica de América Central (RGAC), se ha convertido en un medio de difusión de investigaciones, en las cuales se ha creado un vínculo entre la geología y la arqueología, con el fin de resolver problemas referentes a la vida de las sociedades precolombinas de nuestra región centroamericana.

\section{¿GEOARQUEOLOGÍA O GEOLOGÍA ARQUEOLÓGICA?}

Karl W. Butzer (1982), fue el primero en proponer, desde un punto de vista teórico y metodológico, lo que se conoce como arqueología ambiental o contextual, tomando contexto, en un sentido mucho más amplio del tradicionalmente utilizado en arqueología. Esta arqueología contextual, más interesada en los yacimientos que en los artefactos, se ocupa sobre todo de la expresión multidimensional de la toma de decisiones humanas dentro de un medio natural específico. Y, sin ocuparse directamente de los fenómenos ecológicos, pretende estimular la investigación globalizadora, en base fundamentalmente a las complejas interacciones sistémicas entre los factores y procesos culturales, biológicos y físicos (Butzer, 1982).

Por lo que el objetivo principal de la arqueología ambiental viene a ser definir las características y procesos del ambiente biofísico, susceptibles de suministrar una textura pura y de interactuar con los sistemas socioeconómicos. Un objetivo secundario de este y otros métodos afines, es llegar a comprender el ecosistema humano definido por esa intersección sistémica, es decir, el estudio de los yacimientos arqueológicos o conjuntos de yacimientos, como parte de un ecosistema humano (Butzer, 1982).

Siguiendo el planteamiento hecho por Butzer (1982), una aproximación a la relación que existió entre las sociedades pasadas y su entorno natural, 
es posible llevarlo a cabo a través de estudios en los campos de la arqueobotánica, zooarqueología y geoarqueología. Partiendo de esto, es posible diferenciar las investigaciones que buscan acercarse al entendimiento de la relación ser humano y ambiente, y como lo indica Butzer (1982), vienen a ser interdisciplinarias (se realiza con la cooperación de varias disciplinas), que para el caso de la geoarqueología, necesariamente implica una sinergia tanto a niveles teóricos, metodológicos, como analíticos.

Entonces, y como indica Butzer (1982), geoarqueología implica archaeological research using the methods and concepts of the earth sciences. The term is not synonymous with archaeological geology, and it is not necessarily linked to geology (p. 35). De forma similar, Rapp \& Hill (1998), mencionan que este tipo de investigaciones hacen referencia a la aplicación de cualquier concepto, técnica o conocimiento de las ciencias de la tierra, basado en el estudio de artefactos y procesos involucrados en la creación del registro arqueológico. Nótese la reiteración del término "ciencias de la tierra", lo cual lleva a la arqueología de valerse tanto de métodos y técnicas, que van más allá de la geología, que ya de todas formas son numerosas, por lo que la gama de "lecturas" a las cuales se puede someter el registro arqueológico y su contexto, vienen a engrandecerse.

Y como Butzer (1982) indica, en geoarqueología se hace uso de métodos y técnicas de las ciencias de la tierra, no solamente de geología: ...because the geosciences comprise geography and pedology, as well as geology. Each provides component data essential to the study of environmental systems. The full array of these components includes a formidable list of subfields and composite approaches. Geophysics, geochemistry, stratigraphy, sedimentology, geomorphology, soils, hydrology, climatology, and spatial analysis are all relevant to geo-archaeology in varying degrees (p. 35).

En este punto, es necesaria la definición de dos términos que pueden ser fácilmente confundibles: geoarqueología y geología arqueológica. Según Rapp \& Hill (1998), la diferencia reside en que la geoarqueología tiene como finalidad dar respuesta a un problema arqueológico, es decir, objetivos con relación a cómo fue la vida en sociedades pasadas (tecnologías, medios de producción, respuesta a cambios climáticos, redes de comercio, y otros), o en otras palabras, la búsqueda de una síntesis para contraponer la sociedad y su ambiente. Mientras que por otro lado, geología arqueológica viene a ser todas las investigaciones de índole geológico, que pueden aportar información en los trabajos arqueológicos (el medio para llegar a la síntesis), por ejemplo, mapas geológicos que brinden información en la búsqueda de materias primas para la elaboración de instrumentos líticos en una región específica.

\section{INVESTIGACIONES EN COSTA RICA}

En nuestro país, las investigaciones que pueden ser catalogadas como geoarqueológicas, son reducidas, estas se pueden dividir en dos grandes grupos. Los primeros trabajos son aquellos interesados en la relación de la vida precolombina con la actividad volcánica, donde las interpretaciones giran en torno a un posicionamiento teórico ecologista con tintes deterministas, más o menos definidos. Por otro lado, están los trabajos arqueométricos (caracterización o medición de los materiales arqueológicos a partir de métodos físico y/o químicos), principalmente enfocados en cerámica, y donde resalta el interés por el entendimiento de rutas de comercio, y las interacciones socio-culturales relacionadas con éstas.

\section{Principales temáticas}

\section{Relaciones ambiente - ser humano}

Carlos H. Aguilar (1977), primer arqueólogo costarricense, fue quien realizó lo que se puede catalogar como el primer trabajo geoarqueológico, llevado a cabo por un nacional. Este fue un trabajo de prospección en los alrededores del volcán Arenal, concentrado en el área a ser inundada al cerrarse la represa Sangregado, y a un área marginal afectada por la construcción de un camino construido por el ICE, la mayor parte de ellas actualmente bajo el nivel de las aguas del 
lago Arenal. El estudio nació debido al potencial arqueológico, visto en una investigación previa llevada a cabo por parte de la Smithsonian Institution, y la posible relación con la secuencia de materiales volcánicos, planteada inicialmente por el Dr. William G. Melson, vulcanólogo de la institución, quien luego realizó el levantamiento de la secuencia tefroestratigráfica para la región, para lograr establecer la relación entre las culturas precolombinas y las actividades del volcán Arenal (Aguilar, 1977).

Aguilar (1977) concluye que el área de El Arenal, por sus condiciones ecológicas ofreció condiciones muy favorables para el establecimiento de asentamientos humanos desde períodos precolombinos; la existencia del ser humano data de aproximadamente de 1500 a.C. hasta el momento de la conquista, así como ha podido establecer una secuencia cultural y una relación determinante entre esta secuencia y las unidades de tefra establecida.

Entre los años de 1984 y 1987 se llevaron a cabo una serie de análisis, enmarcados dentro de una investigación multidisciplinaria, El Proyecto Prehistórico Arenal, que incluyó arqueología, vulcanología, pedología, sensores remotos y botánica, en los alrededores de la laguna del volcán Arenal. Se investigaron sitios arqueológicos ubicados principalmente en las orillas del lago Arenal, donde se encontró evidencias de ocupación desde los periodos Paleoindio (18000-8000 a.C.) y Arcaico (8000-2000 a.C.), hasta el momento de la conquista, estableciéndose en 2000 a.C. el inicio de asentamientos en la región del Arenal (Sheets, 1994).

Este proyecto estuvo dirigido hacia el entendimiento del establecimiento y adaptaciones de los habitantes precolombinos en los alrededores de la cordillera de Tilarán (Sheets, 1994), quienes estuvieron inmersos en un proceso cíclico, que envolvió erupciones periódicas del volcán Arenal y la subsecuente formación y recuperación de los suelos y su vegetación. El autor menciona que América Central es un excelente laboratorio para entender la adaptación del ser humano dentro de zonas volcánicas activas, ya que cuenta con diferentes tipos de climas, gran cantidad de volcanes actualmente activos y con un registro que va desde grupos de cazadores-recolectores hasta sociedades estatales estratificadas.

En 1985, dentro del marco del Proyecto Geotérmico Miravalles, se iniciaron una serie de estudios en los alrededores del volcán Miravalles, donde se pretendió adquirir información sobre la historia ocupacional de la región, identificar la distribución de asentamientos humanos, determinar su cronología y reconocer posibles correlaciones con eventos volcánicos, además de conocer la historia vulcanológica de la región. Para luego, eventualmente utilizar la información para estimar niveles de riesgos relacionados con la actividad eruptiva de los volcanes de la región, puesto que podría afectar la seguridad y desarrollo futuros del Proyecto Geotérmico Miravalles (Hurtado y Alvarado, 1988).

Los autores señalan que esta investigación, debido a su carácter interdisciplinario que incorpora información sobre fenómenos biofísicos, debe incrementar las posibilidades de explicación de procesos socioculturales del pasado (Hurtado \& Alvarado, 1988, p. 78). Ejemplo de este alcance, lo constituye el reconocimiento de una toba rojiza, de la formación Aguacate, descrita como culturalmente estéril, en el cual sobreyace el sitio arqueológico Mogote (BM-5), donde se encontraron lascas y fragmentos de piedra tallada, asociadas a fogones, datado para el Arcaico, donde todo el componente fue cubierto en una fecha posterior a la ocupación humana, por una capa de ceniza volcánica que varía en el sitio entre 30 a $60 \mathrm{~cm}$ de grueso. Esta capa, que debe corresponder a una erupción volcánica de fecha no precisada, ha permanecido por un tiempo suficiente como para que se desarrolle un suelo rico en humus en su parte superior (Hurtado \& Alvarado, 1988, p. 82).

\section{Análisis cerámicos}

La búsqueda de evidencias de la manufactura cerámica ha creado interés desde el siglo XVII (Orton et al., 2008), inicialmente los esfuerzos se concentraron en la llamada "alta tecnología", asociada a artefactos considerados de gran calidad. 
Estos estudios se han desarrollado, históricamente, desde dos orientaciones principales: la tecnología vista como indicador de cambio social y la elaboración de trabajos etnográficos, relativos a la comprensión de la producción de artefactos, obtención de recursos, distribución y otros aspectos relacionados. Los análisis de antiguas tecnologías, interesados en la comprensión de los métodos de manufactura (Bronitsky, 1989; Herrera, 2001; Sanhueza et al., 2004; de la Fuente et al., 2005), en campos específicos; como el análisis de engobes, donde interesa, en primera instancia, conocer características como su espesor, inclusiones o desgrasantes (orientación y composición) y etapas de segunda cocción para fijación (condiciones atmosféricas oxidantes, temperatura y tiempo); toda esta información podrá ser aprovechada para el entendimiento de la especialización de la manufactura de artefactos utilizados para el almacenamiento de sustancias líquidas, por mencionar un ejemplo. Herrera (2001: 2) menciona que el estudio de la producción alfarera ha tenido muy poco desarrollo dentro del quehacer arqueológico de Costa Rica, las líneas de trabajo en la investigación cerámica siguen estando relacionadas más frecuentemente con las clasificaciones modales y tipológicas en la búsqueda de la distinción espaciotemporal de las ocupaciones regionales antiguas.

Junto con la elaboración de este tipo de análisis, ha ocurrido la incursión, por parte de los arqueólogos, en disciplinas auxiliares, como la geología, química o física, las cuales han brindado a los investigadores, la utilización de técnicas propias de esos campos, como la elaboración de secciones delgadas, análisis composicionales y de elementos traza, por mencionar algunos.

Sin lugar a dudas, los análisis de procedencia han dominado el interés de los investigadores en nuestro país y en la región mesoamericana. Estos estudios se han centrado, principalmente, en materiales policromos, dentro de Gran Nicoya y en contextos fuera de ésta; las técnicas utilizadas han sido de diversas clases: análisis instrumental por activación de neutrones (Lange et al., 1987; Bishop et al., 1988; 1992; Bishop, 1992-1993; Chapdelain et al., 2008), fluorescencia de rayos X (Salazar \& Moya, 1985), difracción de rayos
X (Accola, 1977), microscopía electrónica MER (Neff, 1990) o SEM y/o análisis petrográficos (Lange et al., 1987). El principal objetivo de estas investigaciones, ha sido la diferenciación entre cerámicas de producciones locales y no locales, a través del establecimiento de perfiles composicionales particulares, que son compartidos por una subserie de artefactos, considerados propios de un sitio o una región (Lange et al., 1987); con el fin de indicar posibles vías y direcciones de redes de comercio e intercambio de los materiales.

\section{Oro y piedra}

Además se deben mencionar los trabajos de Fernández (2001), quién a partir de análisis arqueométricos (Espectroscopía de Fluorescencia de Rayos, Microscopía Electrónica de Barrido y Energía Dispersiva de Rayos X) de las materias primas, tecnología y las relaciones sociales en torno a la metalurgia, en Nicaragua, Costa Rica y Panamá; los datos obtenidos permitieron identificar la utilización de distintas fuentes de materias primas para la fabricación de los objetos, incluyendo el empleo de oros primarios y secundarios, así como cobre nativo de vetas polimetálicas.

Por último Fernández \& Alvarado (2006) caracterizan las técnicas de manufactura, materias primas, y el uso y función que se le dieron a los artefactos líticos, fabricados entre el 300 a.C. y 1500 d.C., he igualmente aportan datos sobre los yacimientos geológicos y el posible aprovechamiento por parte de los pueblos indígenas precolombinos.

\section{Aportes desde la RGAC}

La RGAC, como medio difusor de las investigaciones en geociencias de la región, viene a mostrar parte del interés en las investigaciones involucradas en el campo geoarqueológico. Si bien es cierto, el número de documentos es reducido, algo importante a tomar en cuenta, es que este tipo de trabajos se encuentran difundidos principalmente por revistas especializadas en el campo de la 
arqueología, como American Antiquity, Journal of Archaeological Science, Geoarchaeology o Current Anthropology, donde tanto el número de trabajos como investigadores, han aumentado con el tiempo.

El primer trabajo geoarqueológico llevado a cabo con materiales de Costa Rica fue elaborado por Fischer (1882), con una serie de objetos de piedra verde, localizados en los museos de Bremen. Además de la caracterización de los artefactos costarricenses, mediante la aplicación de una metodología específica para la caracterización de los minerales, en el documento Fischer (1882) también realiza interesantes comparaciones con materiales de otras regiones de mesoamérica, posible uso, función y representatividad, además del planteamiento de la reutilización de artefactos para la elaboración de los objetos que él analizó. Para un análisis exhaustivo del documento, se recomienda la lectura de Hurtado et al. (2007).

Una tendencia moderna en arqueología, es el estudio de los contextos con el mínimo de intervención y tiempo, por lo cual, la aplicación de técnicas geofísicas han demostrado ser una herramienta útil en arqueología, tanto en etapas de prospección como durante la realización de las investigaciones (Fig. 1). Mojica et al. (2006) presentan una prospección realizada en el sitio arqueológico Panamá Viejo, llevada a cabo en el sector central, los rangos de magnitud de las anomalías magnéticas facilitaron la identificación de ciertos rasgos de tipo hispánico y además, la presencia de artefactos metálicos

propios de una época de ocupación más reciente, cuyas profundidades fueron determinadas con la realización de tomografías eléctricas. Para tener un panorama general del estado de la utilización de métodos y técnicas geofísicas en arqueología, Arias \& Mojica (2003) realizan una recopilación de trabajos, donde gracias al análisis de las señales magnéticas, eléctricas y electromagnéticas, registradas en sitios arqueológicos precolombinos y coloniales, se han llevado a cabo exitosamente el estudio del registro arqueológico, así como avances a nivel metodológico.
Obando \& Peraldo (2011), con su evaluación, realizan un interesante aporte en la temática de preservación del patrimonio cultural, en el Monumento Nacional Guayabo, a través de un reconocimiento geológico regional y local (a nivel de sitio). Este es un estudio de las condiciones geológicas fue posible la detección de problemas de inundación, bajos $\mathrm{pH}$, bioturbación en los componentes de las estructuras, y presencia de deslizamientos en las áreas circundantes al sitio. Si bien, y siguiendo las definiciones de Butzer (1982) y Rapport \& Hill (1998), ya antes discutidas, este trabajo no puede ser considerado como geoarqueológico, es indudable su aporte a la temática de preservación, así como muestra los beneficios del cartografiado geológico y geomorfológico a pequeña escala, que en el caso de la arqueología, brinda la mejor información para ser utilizada por los investigadores.

El trabajo de Obando et al. (2011) representa el mejor ejemplo de una investigación geoarqueológica dentro de la RGAC, según la definición aportada a inicios de este escrito. Aquí los autores (geólogos y arqueólogos) realizaron una caracterización petrográfica de fragmentos cerámicas del Clásico Tardío (250-900 d.C.), provenientes del sitio arqueológico maya Chinikihá (Chiapas, México). Las excavaciones y los análisis macroscópicos modales en la zona nuclear, apuntan que Chinikihá es un asentamiento, que durante mediados del siglo IV, parece haber sido un sitio consumidor, aún no se sabe si productor, de cerámicas de texturas burdas, elaboradas con arcillas, cuyos enseres se caracterizan por la presencia abundante de minerales carbonatados. Para el siglo VIII, la cerámica hecha con arcillas de diferentes texturas con cuarzo, son los que predominaron hasta el final de la época de ocupación del asentamiento, tradición cerámica similar a la región de Palenque, por lo que una de las preguntas de la investigación fue el de determinar, las diferencias petrográficas (involucrando la composición, textura, matriz y partículas minerales) de las pastas cerámicas. Los resultados del análisis petrográfico permitieron evidenciar cambios en las fuentes 


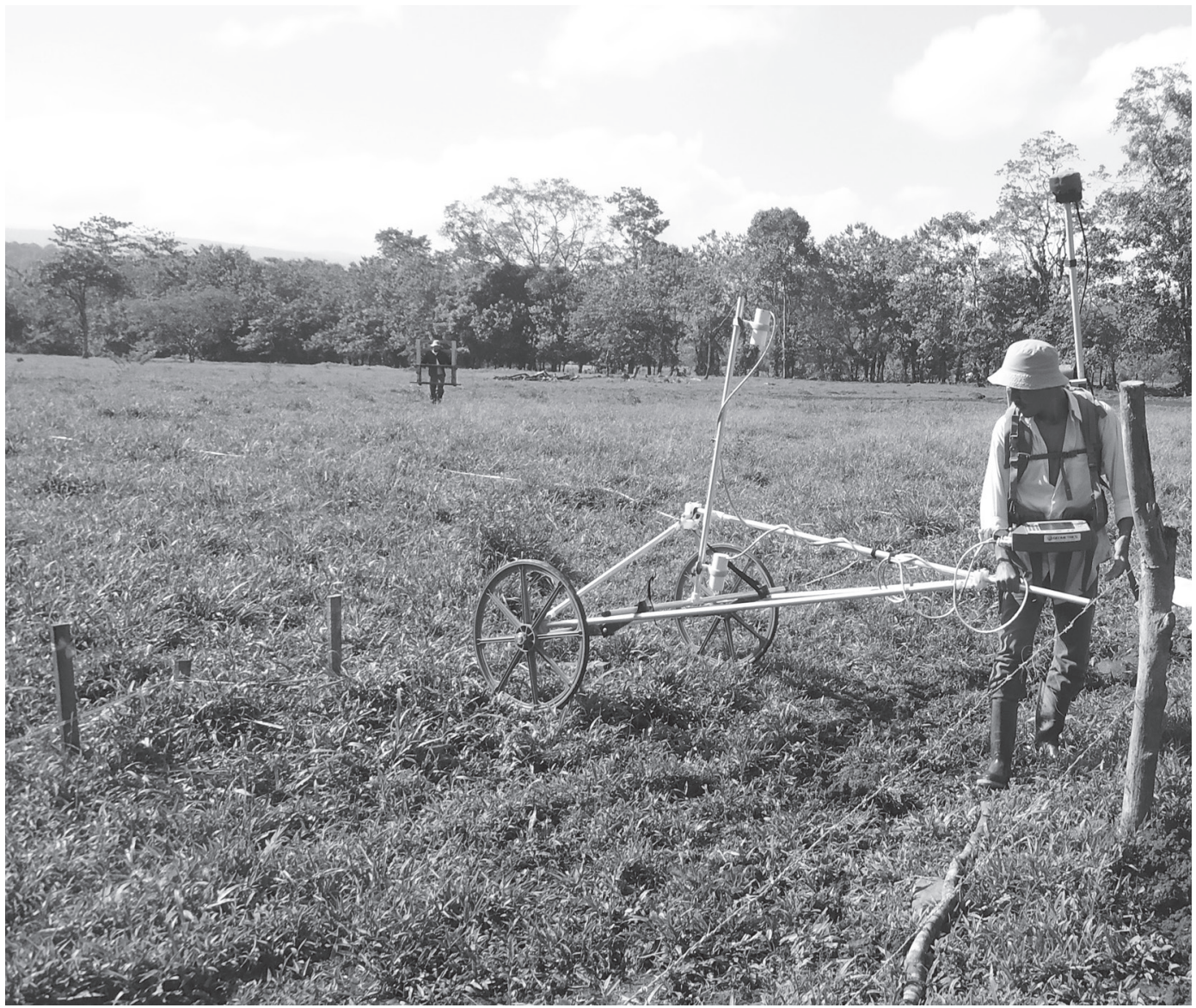

Fig. 1: Prospección geofísica (febrero de 2014), utilizando un magnetómetro de cesio, en un sector del sitio arqueológico Nuevo Corinto (Pococí, Limón, Costa Rica).

de materia prima, para la manufactura cerámica (se pasó de pastas carbonatadas a silicoclásticas), así como su posible ubicación, además de las temperaturas máximas de cocción.

\section{COMENTARIOS FINALES}

Si bien es cierto, que no todas las investigaciones relacionadas con una problemática arqueológica, presentes en la RGAC, pueden ser catalogadas como geoarqueológicas, es imposible de negar que brindan información valiosa en el entendimiento, no solamente de los pueblos precolombinos, sino también, para periodos posconquista, como en el ejemplo de Panamá Viejo.

Las prospecciones geofísicas en sitios arqueológicos, los análisis de proveniencia y caracterización de materias primas, son ejemplos de la clase de temáticas que abarcan los trabajos geoarqueológicos que ha divulgado la RGAC, donde el geólogo brinda una caracterización e interpretación del registro material, a partir del cual los arqueólogos completan sus interpretaciones, y que al final, llegan a ser diferentes a aquellas, formuladas desde los métodos y técnicas tradicionales en arqueología. 
La geoarqueología es un campo interdisciplinario de investigación, por lo que implica un paso más que la mera multidisciplinariedad, es decir, el diseño de tales trabajos debería de expresar la conjunción entre arqueología y geociencias, a niveles teórico, metodológico y claro que en el análisis de los datos. Todo con el fin de llegar a dar respuestas a interrogantes de cómo fue que vivieron sociedades no existentes hoy en día.

Desde una perspectiva geoarqueológica, los análisis cerámicos, por ejemplo, no solamente llevan a caracterizaciones "complejas" de sus componentes. Muchos otros elementos han sido evaluados, a partir del análisis de las tecnologías de producción cerámica, como aspectos referentes a las unidades de producción, conformación, éxito y su papel en el mantenimiento de procesos; el rol de hombres, mujeres y niños dentro de grupos alfareros, la influencia de la conformación de centros de poder o influencia políticos, filiaciones políticas expresadas en la manufactura de los artefactos, cómo se transmitían los conocimientos de elaboración cerámica, o qué procesos mediaban en el aprendizaje de la producción cerámica (Allsworth-Jones, 1996; Bowser, 2000; Crown, 2001). Elementos que pueden ser accesibles, a partir del registro arqueológico, a través del análisis de los procesos y tecnologías de producción, la diferenciación de cambios o continuidad de éstos y sobre todo, por medio de una lectura detallada de los materiales en estudio.

\section{REFERENCIAS BIBLIOGRÁFICAS}

ACCOLA, R., 1977: Análisis de la difracción de rayos $\mathrm{X}$ : $\mathrm{Su}$ aplicación experimental en el estudio de la cerámica policromada de Nicoya, Costa Rica.- Vínculos, 3(1): 37-45.

AGUILAR, C. H., 1977: Introduction to the archeology of the Arenal volcano area: tephrastratigraphy and cultural sequences.Nat. Geograph. Soc. Res. Report, 1977 Projects: 95-107.

ALLSWORTH-JONES, P., 1996: Continuity and change in Yoruba pottery.- Bull. School of Oriental and African
Studies, 59(2): 312-322, doi: 10.1017/ S0041977X00031591.

ARIAS, M. E. \& MOJICA, C. A., 2003: Prospección arqueológica en América Central mediante el uso de métodos geofísicos.- Rev. Geol. Amér. Central, 29: 53-41.

BISHOP, R. L., 1992-1993: Análisis de composición de la cerámica en el sur de América Central.- Vínculos, 18-19(1-2): 9-29.

BISHOP R. L., LANGE, F. W., ABEL-VIDOR, S. \& LANGE, P. C., 1992: Compositional characterization of the Nicaraguan ceramic sample.- En LANGE, F. W., SHEETS, P., MARTÍNEZ, A. \& ABEL-VIDOR, S. (eds): The archaeology of Pacific Nicaragua.- Univ. of New Mexico Press, Albuquerque: 135-162.

BISHOP, R. L., LANGE, F. W. \& LANGE, P. C., 1988: Ceramic paste compositional patterns in Greater Nicoya pottery.- En LANGE, F. W. (ed.): Costa Rican art and archaeology: essays in honor of Frederick R. Mayer.- Johnson Pub., Colorado: 11-44.

BOWSER, B. J., 2000: From pottery to politics: an ethnoarchaeological study of political factionalism, ethnicity, and domestic pottery style in the Ecuadorian Amazon.- J. Archaeol. Method and Theory, 7(3): 219-248.

CHAPDELAINE， C., VÁZQUEZ, R. \& KENNEDY, G., 2008: Análisis de activación neutrónica de cerámica arqueológica del Valle Central y Turrialba, Costa Rica.Vínculos, 31(1-2): 109-132.

CROWN, P. L., 2001: Learning to make pottery in the prehispanic American Southwest.- J. Anthropol. Res. 57(4): 451-469.

FERNÁNDEZ, P., 2011: Metalurgia y relaciones sociales en el sur de América Central (300-1500 d.C.).- 279 págs. Univ. de Costa Rica, San José [Tesis M.Sc.]. 
FERNÁNDEZ, P. \& ALVARADO, G. E., 2006. Artesanos y piedras. Herramientas y escultura precolombina en Costa Rica. Craftsmen \& stones. Pre-Columbian stone tools and sculpture in Costa Rica.128 págs. Fundación Museos del Banco Central, San José.

FISCHER, H., 1882: Informe sobre un número de esculturas de piedra de Costa Rica.- Traducción de LÜCKE, O.H. \& ALVARADO, G.E., 2007 [del original Bericht über eine Anzahl Steinsculpturen aus Costarica.- Abhandlungen herausgegeben vom naturwissenschaftlichen Verein zu Bremen, 7: 153-185].- Rev. Geol. Amér. Central, 37: 45-64.

HERRERA A., (2001): Tecnología alfarera de los grupos ribereños de la cuenca del Golfo de Nicoya durante los periodos Bagaces (300800 d.C.) y Sapoá (800-1350 d.C.).- 226 págs. Univ. de Costa Rica [Tesis Lic.].

HURTADO, L., ALVARADO, G. E., \& LÜCKE, O. H., 2007: El carácter pionero de los trabajos de Heinrich Fischer $(1875,1881$, 1882) sobre los jades sociales en la geoarqueología de Costa Rica.- Rev. Geol. Amér. Central, 37: 35-44.

HURTADO, L. \& ALVARADO, G. E., 1988: Datos arqueológicos y vulcanológicos de la región del volcán Miravalles, Costa Rica.- Vínculos, 14: 77-89.

LANGE, F. W., BISHOP, R.L. Y LANGE, P. C., 1987: La geología y arqueología de la cerámica prehistórica de la Gran Nicoya. Vínculos, 13(1-2): 7-34.

MOJICA, A., ACOSTA, L., GUÉRIN, R., HO, C. A. \& CABALlERO, O., 2006: Geophysical investigations in the Old Panama Archaeological Site.- Rev. Geol. Amér. Central, 34-35: 131-138.
NEFF, E., 1990: Cerámica precolombina, proceso de manufactura de artefactos cerámicos monocromos del valle central Oriental, zona Pejibaye.- 83 págs. Univ. de Costa Rica, San José [Tesis Lic.].

OBANDO, L. G., JIMÉNEZ, S. \& KUSSMAUL, S., 2011: Estudio petrográfico de cerámicas mayas, Clásico Tardío (600-900 d.C.), Chinikihá, Chiapas, México.- Rev. Geol. Amér. Central, 44: 101-118.

OBANDO, L. G. \& PERALDO, G., 2011: Geo-arqueología del Monumento Nacional Guayabo (MNG), Turrialba, Costa Rica.- Rev. Geol. Amér. Central, 45: 119-130.

RAPP, G. JR. \& HILL, C.L., 1998: Geoarchaeology. The earth-science approach to archaeological interpretation.274 págs. Yale University Press, New Haven, Estados Unidos.

RENFREW, C., 1976: Archaeology and the earth sciences.- En DAVIDSON, D. A. \& SHACKLEY, M. L. (eds): Geoarchaeology: Earth science and the past.- Duckworth, Londres: 1-5.

SALAZAR, A. \& MOYA, L.M., 1985: Análisis de cerámica policromada por fluorescencia de rayos $\mathrm{X}$ para estudios arqueológicos. Vínculos, 11(1-2): 101-110.

SHEETS, P. D., 1994: The Proyecto Prehistórico Arenal: an introduction.- En SHEETS, P. D. \& MCKEE, B. R. (eds): Archaeology, volcanism, and remote sensing in the Arenal Region, Costa Rica.- Univ. of Texas Press, Austin: 1-23.

TRIGGER, B. G., 2006: A history of archaeological though.- 730 págs. Cambridge Univ. Press, Cambridge. 
